\title{
DEPRESSÃO INFANTIL PELO OLHAR DA FENOMENOLOGIA ${ }^{1}$
}

\author{
André Luiz dos Santos Lima - UNIFSA \\ Débora de Lima Lira - UNIFSA \\ Tayná de Oliveira Freitas - UNIFSA \\ Renata Madureira Lins de Araújo - UNIFSA ${ }^{2}$
}

\section{RESUMO}

Pensar em depressão é pensar sobre como o corpo e mente apresentam sinais comunicados, reconhecidos e experienciados pelo corpo no qual altera a relação consigo e com o mundo. O presente artigo propõe uma discursão onde como a psicologia tem estudado a infância de acordo com o olhar adulto, baseado na etapa do desenvolvimento humano. Considera-se a depressão uma das doenças que se tornará de maior alcance nos próximos anos, portanto, olhar a depressão agora e para as crianças que sinalizam este adoecimento é olhar de maneira fenomenológica para o problema. Entendemos que a postura reflexiva crítica contribui para a compreensão da depressão e do sujeito infantil no seu mundo vivido. A perspectiva fenomenológica vem contribuir de forma significativa para se estudar e abordar a depressão infantil. Foram realizadas buscas nos portais Scielo, Google Acadêmico e Portal CAPES, no período dos últimos sete anos (2013-2019), o qual foram aceitos 10 artigos. $\mathrm{O}$ estudo demonstra que a depressão é uma patologia que sempre existiu, porém, nunca foi tão focada a crianças e através do olhar do terapeuta baseado na perspectiva humanista fenomenológica possibilitará mudança nesse pensar. Destacamos que a pesquisa aqui apresentada é apenas um retrato para possíveis estudos e pesquisas mais aprofundadas baseado na construção de um saber teórico da realidade.

Palavras-Chave: Depressão Infantil. Fenomenologia. Psicologia.

\section{ABSTRACT}

To think of depression is to think of how body and mind exhibit communicated, acknowledged, and bodyexperienced signals in which they alter their relationship with themselves and the world. This paper proposes a discussion in which psychology studies childhood according to adult appearance based on the stage of human development. Depression is considered one of the diseases that will become more widespread in the coming years. Therefore, to analyze depression now and the children that signal this disease is to look phenomenologically at the problem. It is understood that the critical reflexive posture contributes to the understanding of depression and the child in their lived world. The phenomenological

\footnotetext{
1 Trabalho apresentado no Congresso Brasileiro Ciência e Sociedade (CBCS 2019), promovido pelo Centro Universitário Santo Agostinho, de 03 a 05 de outubro de 2019, em Teresina-PI.

2 Graduanda de Psicologia - 60 Período, UNIFSA. taynafreitas@hotmail.com

Graduando de Psicologia - 6 ${ }^{\circ}$ Período, UNIFSA. andre_lima098@outlook.com

Graduanda de Psicologia $-6^{\circ}$ Período, UNIFSA. deboralira1@ hotmail.com

Graduanda de Psicologia - $6^{\circ}$ Período, UNIFSA. renatalinsaraujo@gmail.com
} 
ANAIS CBCS 2019 | 3 a 5 de outubro de 2019 | Centro Universitário Santo Agostinho - Teresina - PI

perspective contributes significantly to studying and addressing childhood depression. The Scielo, Google Scholar and Portal CAPES portals were searched in the last four years (2014-2018), which analyzed 20 articles. The study demonstrates that depression is a pathology that has always existed but never been so focused on children and, through the therapist's perspective, based on the phenomenological humanist perspective, will allow changes in this thinking. It is emphasized that the research presented here is just a picture of possible further studies and research based on the construction of a theoretical knowledge of reality.

Keywords: Child depression. Phenomenology. Psychology.

\section{INTRODUÇÃO}

Podemos dizer que a depressão consiste em um transtorno que pode afetar o ser humano em seus diferentes estágios da vida. A compreensão de que sujeitos antes da vida adulta também podem ser acometidos por essa enfermidade vem tornando campo de investigação, baseado em dados das últimas décadas, em que alertam para o crescente índice de crianças e adolescentes com este quadro clínico, até então não tanto explorado.

Baseado nas pesquisas para composição deste artigo incluíram que estudos dos últimos sete anos, cada vez mais crianças apresentam sintomatologia depressiva, mostrando também que este assunto diz respeito aos problemas de saúde pública e devem ser tratados com bastante seriedade, afinal, suas consequências podem acompanhar a vida delas por um longo período se não tratadas.

Sabe-se que o papel da criança ao longo da história nem sempre foi o mesmo e por conta disso suas características quanto a sua constituição social, também foram alteradas tornando-as mais adultas. $\mathrm{O}$ consumo cada vez mais excessivo, carregado de um significado cultural, tem provocado estas possíveis mudanças, indicando, assim, o surgimento de uma nova classe etária de crianças adultizadas. Isso também vem mostrar a transformação ocorrida nas relações das crianças com as pessoas da família e sociedade, transformando o estilo, a relação e significação dos objetos. (REIS, 2016)

Segundo Oliveira (2017), no campo da Psicologia esta criança tem sido compreendida a partir do olhar desenvolvimentista, o qual espera que no final ela se torne um adulto realizado. Essa ótica 


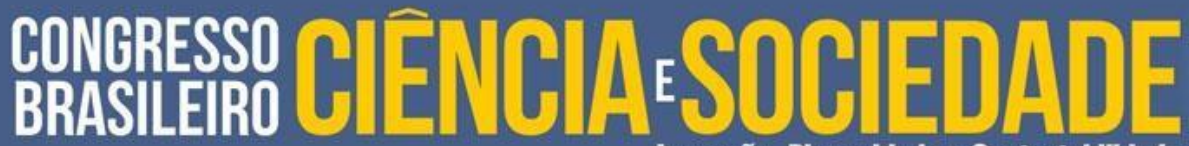 \\ Inovação, Diversiliaile e Sustentahililiadie}

ANAIS CBCS 2019 | 3 a 5 de outubro de 2019 | Centro Universitário Santo Agostinho - Teresina - PI

da psicologia do desenvolvimento nos traz a infância entendida como um estado de passagem precário e efêmero em construção por meio do acúmulo de experiências e conhecimentos.

Portanto, para entender melhor essa relação da depressão infantil pelo olhar fenomenológico este presente artigo vem apresentar uma revisão bibliográfica da literatura com o propósito de discutir como é a psicoterapia em uma perspectiva fenomenológica com a criança que tem sinais de depressão.

\section{METODOLOGIA/MATERIAL E MÉTODOS}

A coleta de dados para a construção deste artigo se deu através de uma revisão bibliográfica da literatura já disponível. Foram pesquisados artigos nas plataformas Scielo, Google Acadêmico, Capes. Como critério de inclusão e exclusão dos artigos, utilizou-se: língua portuguesa, período entre 2013 à 2019 e que abordassem o tema propostos. Aceitos um total de 10 artigos considerados válidos e 12 não atenderam os quesitos da pesquisa. Adotou-se as palavras-chaves como busca: Depressão, Depressão infantil e Fenomenologia.

O objetivo geral desta pesquisa é discutir como a psicoterapia em uma perspectiva fenomenologia atende a criança que tem sinais de depressão.

\section{RESULTADOS E DISCUSSÃO}

\section{DEPRESSÃO}

Podemos chamar esse transtorno da Depressão como um fenômeno, pois tem se destacado bastante na atualidade por sua crescente incidência no mundo todo. De acordo com os últimos dados disponíveis pela Organização Mundial de Saúde (OMS) (2018), o quadro caracterizado de depressão grave está na lista das dez principais causas de doenças a nível mundial, atualmente a principal razão da incapacidade da população. Avaliamos que se esta projeção estiver correta nos 
ANAIS CBCS 2019 | 3 a 5 de outubro de 2019 | Centro Universitário Santo Agostinho - Teresina - PI

próximos vinte anos a depressão subirá neste ranking, se tornando assim a segunda principal causa de doença no mundo. (Santiago,2013).

O diagnóstico da depressão não é fácil, pois demanda avaliação de uma série de sintomas que podem estar associados a outras possíveis patologias. Se direcionado pela Classificação Internacional das Doenças (CID-10) a depressão apresenta uma diferença entre "episódios depressivos" e "transtornos depressivos recorrentes" sinalizando que o que diferencia um do outro é o tempo e frequência em que ocorrem. No Manual Diagnóstico e Estatístico de Transtornos Mentais (DSM-V) o indicativo de orientação para o diagnóstico de depressão se baseia nos seguintes critérios:

“Estado deprimido a maior parte do tempo, anedonia, sensação de culpa ou inutilidade excessivas, dificuldade de concentração, fadiga, distúrbios do sono, agitação ou lentificação psicomotora, aumento ou redução significativa de peso, e ideias recorrentes de morte e suicídio."

Também classifica o quadro pelo número de sintomas em três grupos: "depressão menor" quando apresenta dois ou quatro sintomas com frequência de duas ou mais vezes na semana com estado deprimido ou anedonia, "distimia" somatória de três ou quatro sintomas com no mínimo dois anos de duração incluindo o estado deprimido, "depressão maior" onde há a junção de cinco ou mais sintomas apresentados no período de duas ou mais semanas adicionando o estado de anedonia ou deprimido.

Os sintomas apresentados pela depressão conforme Santiago (2013), inclui a alteração da capacidade de experimentar o prazer, perda de interesse, diminuição da capacidade de concentração, fadiga, sono e alteração no apetite. Podendo alterar também a autoestima e autoconfiança como também apresentar ideias de culpabilidade e/ou indignação. 
ANAIS CBCS 2019 | 3 a 5 de outubro de 2019 | Centro Universitário Santo Agostinho - Teresina - PI

\section{DEPRESSÃO INFANTIL}

Assim como os adultos as crianças por manterem uma relação com a cultura e com o outro, e se realizar no mundo para se tornar adulto, sofrem intensas dificuldades de adaptação durante seu período de formação como humano. Todavia, a criança sempre foi comparada ao adulto e constantemente se percebe esbarrando em novas situações de sua vida. É neste período da infância que o humano está percebendo e tendo relações de transformações com o próprio corpo e com as diversas situações em sua vida.

Pelas inúmeras mutações culturais que as gerações vem sofrendo, as crianças não conseguem mais ser crianças, elas precisam se adaptar rapidamente ao mundo que lhe exige tantas responsabilidades que podem prejudicar o desenvolvimento sadio, e em meio a estes conflitos ou em determinada situação específica, na tentativa de sobreviver ao desencontro consigo mesma, acabem por adoecer na tentativa de informar que algo não vai bem.

É nessa desenfreada tentativa de compreender toda essa remodelagem da construção social que Bolconte (2014) vem nos dizer que o fenômeno da depressão na contemporaneidade se justifica, principalmente, pelo alto nível de sofrimento que tem alimentado e causado ao ser humano, principalmente marcado pela égide do individualismo. Esta ausência de empatia, constitui um sintoma social da depressão.

\section{FENOMENOLOGIA}

Através da possibilidade de mudança deste corpo doente para o corpo sadio que o existencialismo por intermédio da fenomenologia aplicada na psicoterapia busca recursos apropriados para a compreensão deste fenômeno enquanto vivido pelo indivíduo. A trajetória requer a redução fenomenológica, que possibilita penetrar na vivência dessas crianças e ajudá-las. A atitude do terapeuta se pautará na investigação desvestida de todos os seus conceitos e experiências, onde haverá a suspensão ou o abandono dos conceitos e teoria sobre a experiência, porém de forma imprescindível e necessária. 
ANAIS CBCS 2019 | 3 a 5 de outubro de 2019 | Centro Universitário Santo Agostinho - Teresina - PI

Preconizada por Husserl, o "retorno às coisas mesmas" requer este encontro entre o pesquisador com a experiência do sujeito, não como uma representação mais como um fato real, em que ambos são justapostos entre si.

Oliveira (2017), considera que embora o método fenomenológico seja uma ferramenta que auxilie na aproximação da criança e de sua vivência, há na experiência infantil uma certa opacidade para o adulto. Pois, assim classifica-o como desafiadora, tendo que estudar a criança em sua condição própria, mesmo sendo adultos. Aponta que para Husserl a criança não apresenta diferenças em relação ao adulto no quesito habilidades de percepção, contudo, há um ideal e um progresso a ser atingido ao longo da infância para que a criança possa despertar para o mundo humano, tendo assim seu primeiro ato de empatia.

Na busca de considerar um cuidado mais adequado a essa criança, Santiago (2013) aponta que:

\footnotetext{
“A psicologia fenomenológica-existencial é uma abordagem que traz uma compreensão diferenciada dos fenômenos psicopatológicos, em especial quando comparada aos modelos tradicionalmente focados em aspectos biológicos e fisiológicos, centrados numa intervenção nosológica e farmacológica."
}

A escuta do terapeuta sobre os sinais do corpo deprimido pode revelar tanto a depressão como o sujeito que a vive, é preciso compreender o corpo como um mediador de um mundo e essa compreensão tem um sentido.

Vale ressaltar que embora Carl Rogers não seja autor da Fenomenologia, pois é criador da Abordagem Centrada na Pessoa, se aproxima da ideia fenomenológica, no qual pela sua ótica entende a criança como alguém capaz de tomar decisões em relação com o outro e com o mundo, exercendo uma condição de existência da mesma forma que o adulto é bastante válida. A autonomia apontada por esta criança como uma pessoa aberta pode torná-la pessoa diante dela mesma e viver pronta para suas experiências. Assim como uma criança, Carl Rogers nos diz que o 
ANAIS CBCS 2019 | 3 a 5 de outubro de 2019 | Centro Universitário Santo Agostinho - Teresina - PI

ser humano encontra-se em um processo contínuo e em constante atualização rumo a sua autonomia. (Oliveira, 2017).

$\mathrm{Na}$ tentativa de compreender os fatores que levou esta criança a se encontrar neste quadro clínico específico de depressão, podemos citar a irritabilidade com alterações de humor, angústia, ansiedade, sinais de inquietação e agressividade, entendida muitas vezes pela dificuldade de lidar com os sentimentos, solidão, queda no desempenho escolar, alteração no sono, problemas familiares, abuso sexual, fobias, falecimento de algum membro da família, dentre outros. Observa-se que quanto maior o número de sintomas constatados e menor a idade da criança mais complexo tornase o diagnóstico de depressão. Muitas vezes as crianças pequenas não conseguem reconhecer e comunicar o que está acontecendo.

Como o corpo é eminentemente um espaço expressivo podemos destacar o desequilíbrio vivido entre o corpo-sujeito e o corpo-objeto. Através da ferramenta de escuta e observação, assim como na fala pode-se perceber está falta de conexão entre o Eu e o mundo. A depressão pode ser descrita a partir do reconhecimento de sinais do corpo e da divisão entre o corpo físico e mente.

As dimensões afetivas e psicológicas se destacam nesta análise. No contexto atual da sociedade e da família onde há união transitória entre casais, a violência e o alcoolismo corroboram para aparição da depressão principalmente nesta fase infantil. A estrutura familiar deficiente também comprova este elo, eventos como:

\begin{abstract}
“[...] mal relacionamento com pais e irmãos, ausência ou pouca supervisão familiar, fraco apoio emocional e baixa interação positiva. A ocorrência de eventos estressantes na família também associam-se a depressão, entre os demais problemas como separação dos pais, novo casamentos dos pais, severa violência física cometida pelo pai e pela mãe [...]" (MELO,2018)
\end{abstract}

Melo (2018), enfatiza em sua pesquisa que a dificuldade nas relações podem ser influenciadas por um histórico de depressão materna. Que o bom desenvolvimento do contexto familiar possibilita um ambiente de proteção e tranquilidade as atividades diárias minimizando os demais fatores desencadeantes da depressão. 
ANAIS CBCS 2019 | 3 a 5 de outubro de 2019 | Centro Universitário Santo Agostinho - Teresina - PI

O processo terapêutico em alguns casos, principalmente quando envolve crianças, deve abranger seus membros familiares, na tentativa de compreender que ambiente está criança encontra-se inserida e qual a melhor forma de intervenção a ser aplicada. Os pais exercem grande influência sobre as crianças, portanto, abrange-los aumenta a possiblidade de autorregular a família por completo, assim informando, orientando e sensibilizando a todos sobre o processo depressivo o qual a criança se encontra.

Reis (2016) orienta que diante da dificuldade que a criança encontra em aprofundar-se sobre seus sentimentos a respeito de sua experiência vivida, o psicoterapeuta deve usar algumas técnicas ou ferramentas, sempre com base na relação dialógica com o cliente, evitando que se transforme em exercícios ou recreação pré-estabelecidas, já que estamos tratando de crianças. O objetivo deve ser sempre de estreitar vínculos e de iniciar, progressivamente, o processo de ampliação de sua awareness.

\section{CONSIDERAÇÕES FINAIS/CONCLUSÕES}

Podemos dizer que a verdadeira depressão é aquela que bloqueia a comunicação vital com o mundo, ocorre uma impotência vital de agir, ancorada com um espaço vazio, sem relevo e sem expectativa. A psicoterapia baseada na fenomenologia visa resgatar através de suas técnicas este ser sadio, atento as suas interrupções de contato e seus modos de adoecimento, possibilitando a construção de pontes para o corpo mais expressivo possível.

Espera-se que este estudo possa servir como um novo recorte da necessidade de realização de novas reflexões teóricas acerca do tema proposto, depressão infantil e fenomenologia.

É importante ressaltar que esta revisão bibliográfica anunciou alguns pontos que ainda necessitam de um maior aprofundamento, baseado em pesquisas mais longitudinais dentro do nosso contexto brasileiro. O desenvolvimento em pesquisa sobre depressão infantil é de grande relevância para o cenário acadêmico pois nos traz novas formas de discursões enquanto acadêmicos e futuros profissionais. 
ANAIS CBCS 2019 | 3 a 5 de outubro de 2019 | Centro Universitário Santo Agostinho - Teresina - PI

Por fim, esses achados aqui demonstrados tornam-se mais uma fonte de leitura e reflexões sobre o tema proposto, onde os números iniciais mostram que é crescente o adoecimento desse nicho de sujeitos. Portanto, compreender as diversas formas e expressões que a depressão se constitui é oportunizar um espaço onde os profissionais da saúde, principalmente psicólogos, podem captar melhor o mundo e os significados que a realidade nos objetiva, assim como compreender as condições de possibilidades desta experiência.

\section{REFERÊNCIAS}

BOLCONTE, lara Soares. Perfil do público das clínicas-escola de psicologia do Brasil: uma revisão. Universidade Estadual da Paraíba. Campina Grande - PB, 2014. Disponível em: http://dspace.bc.uepb.edu.br/ispui/bitstream/123456789/8250/1/PDF\%20-

\%20lara\%20Soares\%20Bolconte.pdf

CAMPO, Carla Leal Cinha; Depressão infantil no âmbito da psicologia. Universidade do extremo Sul Catarinense - UNESC. Criciúma, 2013. Disponível em: http://repositorio.unesc.net/handle/1/2140

COLOMBO, É. R. (2018). Ateliê de desenho de livre-expressão com crianças acolhidas: um diálogo entre clínica e fenomenologia. Master's Dissertation, Instituto de Psicologia, University São Paulo, São Paulo. Doi 10.606/D.47.2018. tde -13112018-190536. Retrieved 2019-09-08. Disponível em: http://www.teses.usp.br/teses/disponiveis/47/47133/tde-13112018-190536/en.php

ETAPECHUSK, Jéssica. Fernandes, Luciana Raposo dos Santos. Depressão sob o olhar gestáltico. Psicologia PT. ISSN 1646-6977. Amazonas. $2018 . \quad$ Disponível em: https://www.psicologia.pt/artigos/textos/A1171.pdf

HUTTEL, Joseane et al. A depressão infantil e suas formas de manifestação. Psicologia Argumento, [S.I.], v. 29, n. 64, nov. 2017. ISSN 1980-5942. Disponível em:

<https://periodicos.pucpr.br/index.php/psicologiaargumento/article/view/19659>. Acesso em: 08 set. 2019.

MELO, Anna Karynne; SOUZA, Camila; BRITO, Rosa; PITA, Juliana; MOREIRA, Virginia; O Método fenomenológico em pesquisas de estudo de caso clínico. Investigação Qualitativa em saúde. Atas CIAIQ2018. Fortaleza, 2018. Disponível em: https://proceedings.ciaiq.org/index.php/ciaiq2018/article/view/1868

PEREIRA, Pedro Miguel Domingues Rocha. A temporalidade na perturbação depressiva: segundo a psicopatologia fenomenológica. Instituto de Ciências Biomédicas Abel Salazar, Universidade do 


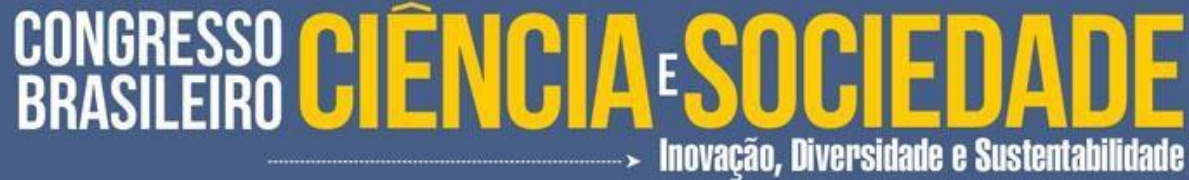

ANAIS CBCS 2019 | 3 a 5 de outubro de 2019 | Centro Universitário Santo Agostinho - Teresina - PI

Porto Mestrado Integrado em Medicina. 2019. Disponível em: https://repositorioaberto.up.pt/bitstream/10216/121505/2/344136.pdf

REIS, Laudeth Alves dos. O ser criança na educação infantil: o desvelar do discurso docente. 2016. 120f. Dissertação (Mestrado em Educação) - Programa de Pós-Graduação em Educação, Universidade Federal do Triângulo Mineiro, Uberaba, 2016. Disponível em: http://bdtd.uftm.edu.br/handle/tede/456

Santiago, Anielli, \& Holanda, Adriano Furtado. (2013). Fenomenologia da depressão: uma análise da produção acadêmica brasileira. Revista da Abordagem Gestáltica, 19(1), 38-50. Recuperado em 08 de setembro de 2019, de http://pepsic.bvsalud.org/scielo.php?script=sci_arttext\&pid=S1809$68672013000100006 \& \operatorname{lng}=$ pt\&tlng=pt.

Oliveira, Elisa Smile Teixeira de, Rosa, Amanda Acco, \& Freitas, Joanneliese de Lucas. (2017). Revisão bibliográfica das publicações acadêmicas sobre a criança na perspectiva fenomenológica. Revista da Abordagem Gestáltica, 23(3), 362-371. Recuperado em 08 de setembro de 2019. Disponível em: http://pepsic.bvsalud.org/scielo.php?script=sci abstract\&pid=S1809-

$\underline{68672017000300012 \& \operatorname{lng}=p t \& n r m=i s o}$ 